Louisiana State University

LSU Digital Commons

$12-10-2011$

\title{
The circumstellar environment of R Coronae Borealis: White dwarf merger or final-helium-shell flash?
}

\author{
Geoffrey C. Clayton \\ Louisiana State University \\ Ben E.K. Sugerman \\ Goucher College \\ S. Adam Stanford \\ Lawrence Livermore National Laboratory \\ B. A. Whitney \\ Space Science Institute \\ J. Honor \\ University of Wisconsin-Madison
}

See next page for additional authors

Follow this and additional works at: https://digitalcommons.Isu.edu/physics_astronomy_pubs

\section{Recommended Citation}

Clayton, G., Sugerman, B., Adam Stanford, S., Whitney, B., Honor, J., Babler, B., Barlow, M., Gordon, K., Andrews, J., Geballe, T., Bond, H., De Marco, O., Lawson, W., Sibthorpe, B., Olofsson, G., Polehampton, E., Gomez, H., Matsuura, M., Hargrave, P., Ivison, R., Wesson, R., Leeks, S., Swinyard, B., \& Lim, T. (2011). The circumstellar environment of R Coronae Borealis: White dwarf merger or final-helium-shell flash?.

Astrophysical Journal, 743 (1) https://doi.org/10.1088/0004-637X/743/1/44

This Article is brought to you for free and open access by the Department of Physics \& Astronomy at LSU Digital Commons. It has been accepted for inclusion in Faculty Publications by an authorized administrator of LSU Digital Commons. For more information, please contact ir@lsu.edu. 


\section{Authors}

Geoffrey C. Clayton, Ben E.K. Sugerman, S. Adam Stanford, B. A. Whitney, J. Honor, B. Babler, M. J. Barlow, K. D. Gordon, J. E. Andrews, T. R. Geballe, Howard E. Bond, O. De Marco, W. A. Lawson, B. Sibthorpe, G. Olofsson, E. Polehampton, H. L. Gomez, M. Matsuura, P. C. Hargrave, R. J. Ivison, R. Wesson, S. J. Leeks, B. M. Swinyard, and T. L. Lim 


\title{
THE CIRCUMSTELLAR ENVIRONMENT OF R CORONAE BOREALIS: WHITE DWARF MERGER OR FINAL-HELIUM-SHELL FLASH?*
}

\author{
Geoffrey C. Clayton $^{1}$, Ben E. K. Sugerman ${ }^{2}$, S. Adam Stanford ${ }^{3}$, B. A. Whitney ${ }^{4,5}$, J. Honor ${ }^{5}$, B. Babler ${ }^{5}$, \\ M. J. Barlow ${ }^{6}$, K. D. Gordon ${ }^{7}$, J. E. Andrews ${ }^{1}$, T. R. Geballe ${ }^{8}$, Howard E. Bond ${ }^{7}$, O. De Marco ${ }^{9}$, W. A. Lawson ${ }^{10}$, \\ B. Sibthorpe ${ }^{11}$, G. Olofsson ${ }^{12}$, E. Polehampton ${ }^{13,14}$, H. L. GomeZ ${ }^{15}$, M. MatsuUra ${ }^{7,16}$, P. C. Hargrave ${ }^{15}$, R. J. Ivison ${ }^{17}$, \\ R. Wesson ${ }^{7}$, S. J. Leeks ${ }^{13}$, B. M. SWINYARD ${ }^{13}$, AND T. L. Lim ${ }^{13}$ \\ ${ }^{1}$ Department of Physics \& Astronomy, Louisiana State University, Baton Rouge, LA 70803, USA; gclayton@ @ phys.lsu.edu, jandrews@phys.lsu.edu \\ ${ }^{2}$ Department of Physics and Astronomy, Goucher College, 1021 Dulaney Valley Rd., Baltimore, MD 21204, USA; ben.sugerman@goucher.edu \\ ${ }^{3}$ IGPP, Lawrence Livermore National Laboratory, Livermore, CA 94551, USA; stanford@ physics.ucdavis.edu \\ ${ }^{4}$ Space Science Institute, 4750 Walnut St. Suite 205, Boulder, CO 80301, USA; bwhitney@ spacescience.org \\ ${ }^{5}$ Department of Astronomy, 475 North Charter St., University of Wisconsin, Madison, WI 53706, USA; jhonor@ astro.wisc.edu, brian@astro.wisc.edu \\ ${ }^{6}$ Department of Physics and Astronomy, University College London, Gower Street, London WC1E 6BT, UK; \\ mjb@star.ucl.ac.uk,mikako@star.ucl.ac.uk, rwesson@star.ucl.ac.uk \\ ${ }^{7}$ STScI, 3700 San Martin Dr., Baltimore, MD 21218, USA; bond@stsci.edu, gordon@ stsci.edu \\ ${ }^{8}$ Gemini Observatory, 670 N. A'ohoku Place, Hilo, HI 96720, USA; tgeballe@ gemini.edu \\ ${ }^{9}$ Department of Physics, Macquarie University, Sydney, NSW 2109, Australia; orsola@ science.mq.edu.au \\ ${ }^{10}$ School of PEMS, University of New South Wales, ADFA, P.O. Box 7916, Canberra, ACT 2610, Australia; w.lawson@adfa.edu.au \\ ${ }^{11}$ Max-Planck-Institut für Astronomie, Königstuhl 17, D-69117 Heidelberg, Germany; bruce.sibthorpe @ stfc.ac.uk \\ ${ }^{12}$ Department of Astronomy, Stockholm University, AlbaNova University Center, Roslagstullsbacken 21, SE-10691 Stockholm, Sweden; olofsson@ astro.su.se \\ ${ }^{13}$ Space Science and Technology Department, Rutherford Appleton Laboratory, Didcot OX11 0QX, UK; \\ edward.polehampton@stfc.ac.uk, sarah.leeks@stfc.ac.uk, bruce.swinyard@stfc.ac.uk, tanya.lim@stfc.ac.uk \\ ${ }^{14}$ Institute for Space Imaging Science, University of Lethbridge, Lethbridge, Alberta T1J 1B1, Canada \\ ${ }^{15}$ School of Physics and Astronomy, Cardiff University, 5 The Parade, Cardiff, Wales CF24 3YB, UK; haley.gomez@astro.cf.ac.uk, p.hargrave@astro.cf.ac.uk \\ ${ }^{16}$ MSSL, UCL, Holmbury St. Mary, Dorking RH5 6NT, UK \\ ${ }^{17}$ UK Astronomy Technology Centre, ROE, Blackford Hill, Edinburgh EH9 3HJ, UK; rji@ roe.ac.uk \\ Received 2011 September 19; accepted 2011 October 14; published 2011 November 21
}

\begin{abstract}
In 2007, R Coronae Borealis ( $\mathrm{R} \mathrm{CrB}$ ) went into a historically deep and long decline. In this state, the dust acts like a natural coronagraph at visible wavelengths, allowing faint nebulosity around the star to be seen. Imaging has been obtained from 0.5 to $500 \mu \mathrm{m}$ with Gemini/GMOS, Hubble Space Telescope/WFPC2, Spitzer/MIPS, and Herschel/SPIRE. Several of the structures around $\mathrm{R} \mathrm{CrB}$ are cometary globules caused by wind from the star streaming past dense blobs. The estimated dust mass of the knots is consistent with their being responsible for the $\mathrm{R} \mathrm{CrB}$ declines if they form along the line of sight to the star. In addition, there is a large diffuse shell extending up to $4 \mathrm{pc}$ away from the star containing cool $25 \mathrm{~K}$ dust that is detected all the way out to $500 \mu \mathrm{m}$. The spectral energy distribution of $\mathrm{R} \mathrm{CrB}$ can be well fitted by a $150 \mathrm{AU}$ disk surrounded by a very large diffuse envelope which corresponds to the size of the observed nebulosity. The total masses of the disk and envelope are $10^{-4}$ and $2 M_{\odot}$, respectively, assuming a gas-to-dust ratio of 100 . The evidence pointing toward a white dwarf merger or a final-helium-shell flash origin for $\mathrm{R} \mathrm{CrB}$ is contradictory. The shell and the cometary knots are consistent with a fossil planetary nebula. Along with the fact that $\mathrm{R} \mathrm{CrB}$ shows significant lithium in its atmosphere, this supports the final-helium-shell flash. However, the relatively high inferred mass of $\mathrm{R} \mathrm{CrB}$ and its high fluorine abundance support a white dwarf merger.
\end{abstract}

Key words: dust, extinction - stars: evolution - stars: mass-loss

Online-only material: color figures

\section{INTRODUCTION}

$\mathrm{R}$ Coronae Borealis $(\mathrm{R} \mathrm{CrB})$ was one of the first variable stars identified and its brightness variations have been monitored since its discovery over 200 years ago (Pigott \& Englefield 1797). The RCB stars form a small group of carbon-rich supergiants which are defined by extreme hydrogen deficiency and unusual variability, characterized by large declines of $8 \mathrm{mag}$ or more due to the formation of carbon dust at irregular intervals (Clayton 1996). Two evolutionary scenarios have been suggested, a double-degenerate white dwarf merger or a finalhelium-shell flash (e.g., Webbink 1984; Clayton et al. 2007). The unexpected discovery that some of the RCB stars have

\footnotetext{
* Based in part on observations with the NASA/ESA Hubble Space Telescope obtained at the Space Telescope Science Institute, and from the data archive at STScI, which are operated by the Association of Universities for Research in Astronomy, Inc., under NASA contract NAS5-26555.
}

isotopic abundances of ${ }^{18} \mathrm{O}$ which are up to 500 times those seen in other stars has increased the likelihood that these rare stars are the result of a white dwarf merger (Clayton et al. 2005, 2007; García-Hernández et al. 2010).

RCB stars have long been known to show IR excesses due to warm circumstellar dust (Clayton 1996; Feast et al. 1997, and references therein). Spectropolarimetry of R CrB obtained in a deep decline showed optical depth effects with wavelength that are consistent with a bipolar geometry with a thick disk or torus obscuring the star and additional more diffuse dust around the poles (Stanford et al. 1988; Clayton et al. 1997). Resolved nebulosity has been detected from the UV to the IR around several RCB stars, including $\mathrm{R} \mathrm{CrB}$ itself, ranging in size from a few arcseconds (V854 Cen, UW Cen) to arcminutes (R CrB, RY Sgr, V CrA, UW Cen) (Walker 1985, 1986; Rao \& Nandy 1986; Pollacco et al. 1991; Clayton et al. 1999; Clayton \& 


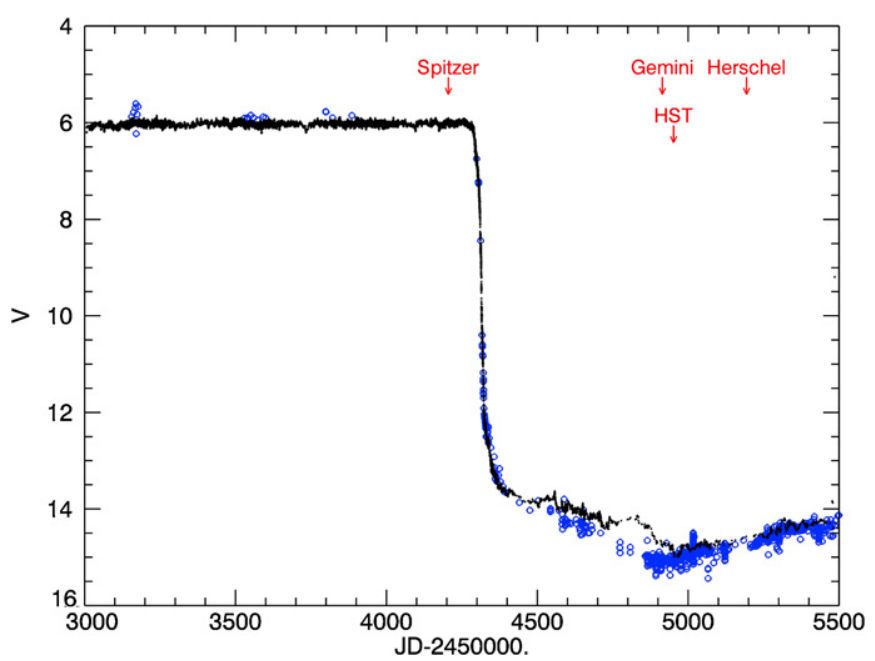

Figure 1. AAVSO data for R CrB since 2004. Visual magnitudes are plotted as black dots. Johnson $V$ data are plotted as blue open circles. The epochs at which the Spitzer, Gemini, HST, and Herschel data were obtained are marked. (A color version of this figure is available in the online journal.)

Table 1

New Observations

\begin{tabular}{lccc}
\hline \hline Date & Telescope & Instrument & Filters \\
\hline 2007 Apr 14/15 & Spitzer & MIPS & $24,70,160 \mu \mathrm{m}$ \\
2009 Mar 23 & Gemini-S & GMOS & $g^{\prime}, r^{\prime}, i^{\prime}, z^{\prime}$, CaT \\
2009 Apr 29 & HST & WFPC2 & F555W, F814W \\
2009 Dec 27 & Herschel & SPIRE & $250,350,500 \mu \mathrm{m}$ \\
\hline
\end{tabular}

Ayres 2001), possibly linking these stars with final-helium-shell flash stars and the central stars of planetary nebulae $(\mathrm{PNe})$. In particular, $\mathrm{R} \mathrm{CrB}$ has a far-IR shell which is $\sim 20^{\prime}$ in diameter, corresponding to $8 \mathrm{pc}$ at its assumed distance (Gillett et al. 1986). More recent observations have used interferometry and adaptive optics to obtain information about the distribution of dust around RCB stars on small spatial scales of tens of AU (Ohnaka et al. 2003; de Laverny \& Mékarnia 2004; Leão et al. 2007; Bright et al. 2011).

In 2007 July, R CrB went into a historically deep and long decline reaching $V=15$. In this state, $\mathrm{R} \mathrm{CrB}$ acts as a natural coronagraph, as the thick dust cloud in front of the star eclipses its photosphere but not its circumstellar material. In this paper, we present new Gemini/GMOS, Hubble Space Telescope (HST)/WFPC2, Spitzer/MIPS, and Herschel/SPIRE images of $\mathrm{R} \mathrm{CrB}$ obtained just before or during this decline, giving wavelength coverage from 0.5 to $500 \mu \mathrm{m}$. We will investigate the morphology and nature of the dust clouds seen near R CrB as well as its large far-IR shell.

\section{OBSERVATIONS AND DATA REDUCTION}

The new observations obtained for this study are summarized in Table 1. Figure 1 shows the recent AAVSO light curve of $\mathrm{R} \mathrm{CrB}$ with the epochs of the various observations marked.

$\mathrm{R}$ CrB was observed with Gemini-S/GMOS on 2009 March 23. Images were obtained in the $g^{\prime}, r^{\prime}, i^{\prime}, z^{\prime}$, and CaT filters. We obtained 8 integrations of $30.5 \mathrm{~s}$ in each of the $g^{\prime}$ and $r^{\prime}$ filters for a total of $244 \mathrm{~s}$, and 14 integrations of $30.5 \mathrm{~s}$ each in the $i^{\prime}, z^{\prime}$, and CaT filters for a total of $427 \mathrm{~s}$. We used the $2 \times 2$ binning and slow readout mode. The field is $6^{\prime} \times 6^{\prime}$. The central part of the field is shown in Figure 2. All images have been geometrically registered to within 0.1 pixel rms. A point-spread

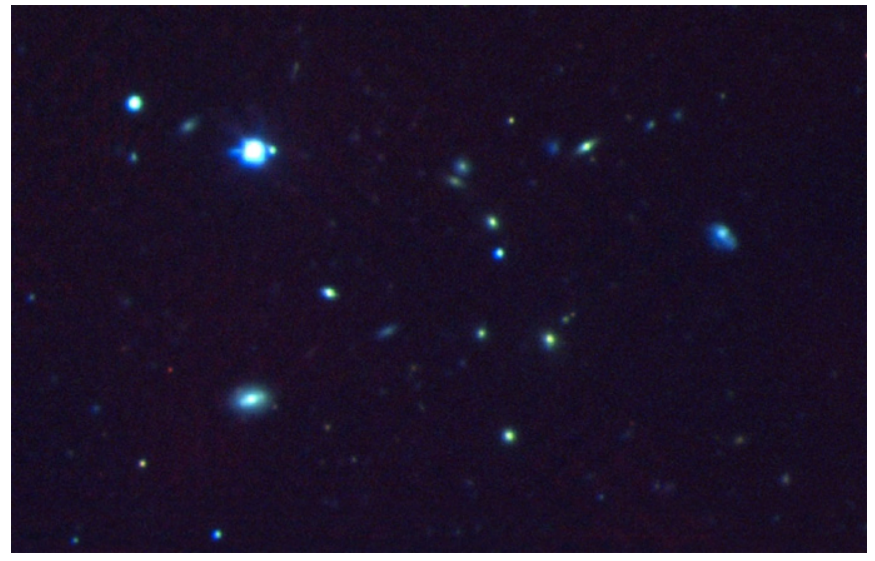

Figure 2. Gemini/GMOS color composite image with $g^{\prime}$ (blue), $r^{\prime}$ (green), and $z^{\prime}$ (red), showing the large number of galaxies near $\mathrm{R} \mathrm{CrB}$ which is the bright object in the upper left. The field is $2^{\prime} \times 2^{\prime}$. North is up and east to the left.

(A color version of this figure is available in the online journal.)

function (PSF) centered on $\mathrm{R} \mathrm{CrB}$ has been subtracted where the sky brightness has been ramped down to zero as one goes out 27 pixels in radius. The plate scale is $0{ }^{\prime} 146$ pixel $^{-1}$. The seeing was $\sim 0^{\prime}$. 8 . These PSF-subtracted images are shown in Figure 3.

R CrB was observed with HST/WFPC2 on 2009 April 27. Images were obtained with the F555W and F814W filters with total integration times of $487 \mathrm{~s}$ and $496 \mathrm{~s}$, respectively. The individual images were stacked and drizzled. The images were then registered and a PSF was subtracted. These images are shown in Figure 4. WFPC2 images of R CrB taken on 1996 July 1 (JD 2450265) with the F469N filter were downloaded from the MAST (Multimission Archive at STScI) archive (Ueta et al. 2000). The exposures were $4 \times 20 \mathrm{~s}$ and $2 \times 400 \mathrm{~s}$. When these images were obtained $\mathrm{R} \mathrm{CrB}$ was at a visual magnitude of $\sim 8.1$ (AAVSO) while recovering toward maximum light after a deep decline. The individual images were stacked and drizzled.

A $U B V R I$ photometric sequence has been established for the R CrB field (Landolt \& Clem 2010). A number of stars (nos. $13,14,19,20,22,23,31,34,35,37,39)$ from that sequence are also in the Gemini/GMOS field. Two of the stars (nos. 20, 22) lie in the HST/WFPC2 field but none are on the PC chip. In addition, a strip from the Sloan Digital Sky Survey (SDSS) telescope crosses R CrB. Several of the photometric sequence stars (nos. 13, 14, 19, 20, 23, 35, 37, 39) from Landolt \& Clem (2010) also have ugriz photometry ${ }^{18}$ and lie in the GMOS field.

Spitzer/MIPS scan maps were obtained of R $\mathrm{CrB}$ at 24, 70, and $160 \mu \mathrm{m}$ on 2007 April 14/15 as part of program 30029. We mapped a $20^{\prime}$ wide area using 15 legs at the medium scan rate with $80^{\prime \prime}$ (1/4 array) cross-scan offsets. The total integration time was $15,536 \mathrm{~s}$. R CrB was observed twice to allow for good germanium (70 and $160 \mu \mathrm{m})$ transient and asteroid removal. The second epoch maps allow for the clean removal of the germanium responsivity drifts which are especially important for mapping extended emission around $\mathrm{R} \mathrm{CrB}$. The scan maps were reduced and mosaiced using the MIPS Data Analysis Tool (Gordon et al. 2005) supplemented with custom reduction scripts written specifically to improve the MIPS reductions of extended sources. The custom reduction scripts include extra steps beyond that of the MIPS DAT. At $24 \mu \mathrm{m}$, the extra steps include readout offset correction, array averaged

\footnotetext{
18 http://www.sdss.org/
} 

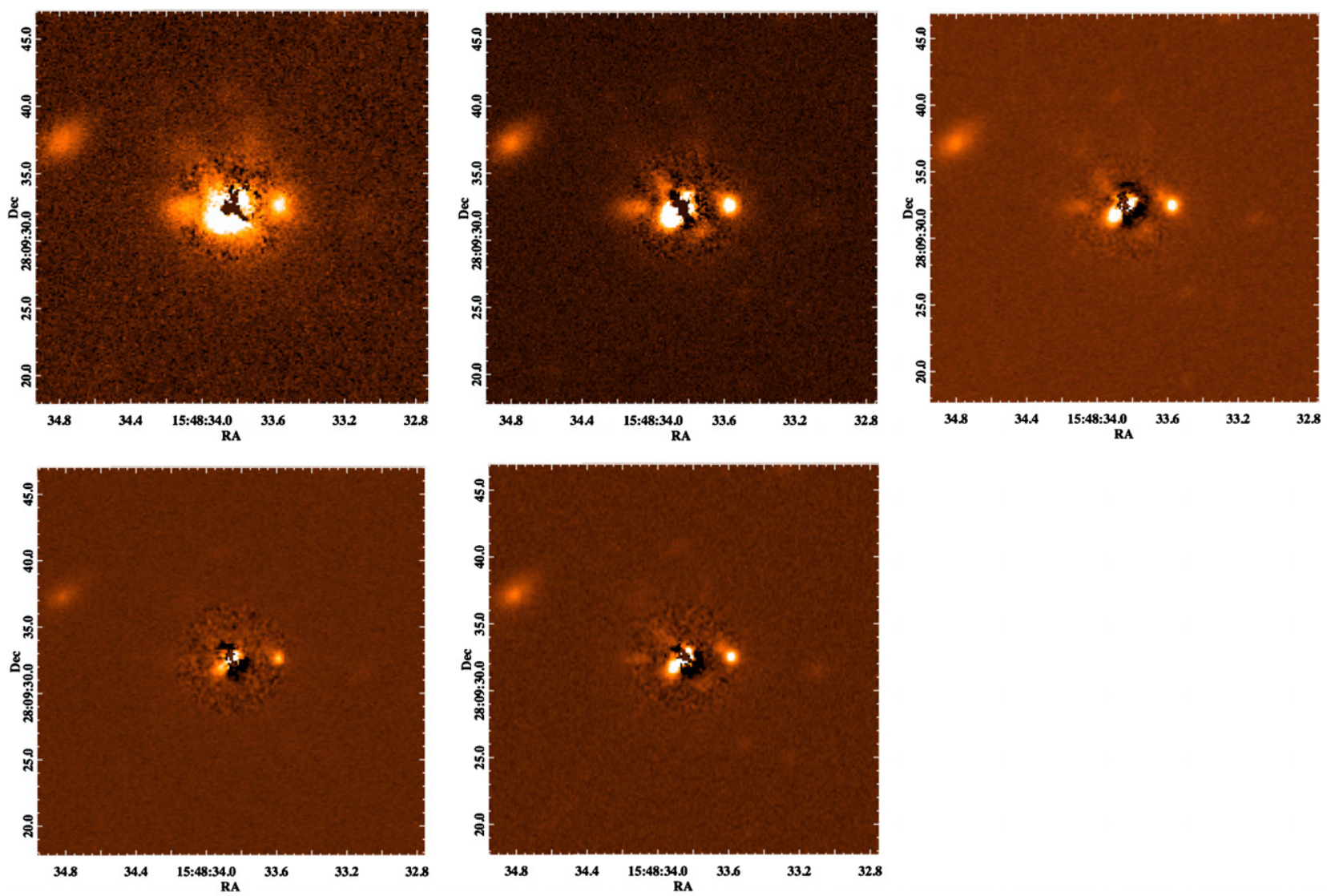

Figure 3. Gemini/GMOS $g^{\prime}, r^{\prime}, i^{\prime}, z^{\prime}$, and CaT images. A stellar PSF has been subtracted from the position of R CrB for each image. There is a faint star $3^{\prime \prime}$ west of $\mathrm{R} \mathrm{CrB}$ and a galaxy about $15^{\prime \prime}$ northeast of $\mathrm{R} \mathrm{CrB}$. North is up and east to the left.

(A color version of this figure is available in the online journal.)
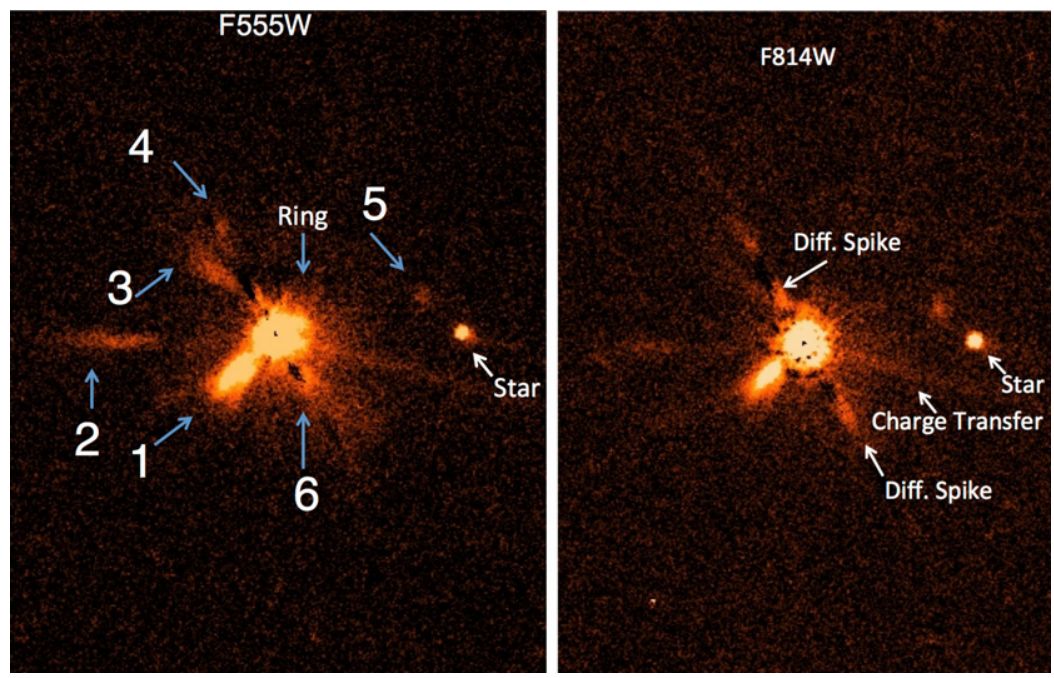

Figure 4. HST/WFPC2 F555W (left) and F814W (right) images with a PSF removed from the position of R CrB. White arrows indicate instrumental features and the companion star. Blue arrows indicate real nebular structures around $\mathrm{R} \mathrm{CrB}$. The angular distance between $\mathrm{R} \mathrm{CrB}$, which lies in the center of the images, and the faint star to the west is $\sim 3^{\prime \prime}$. The cometary knots are numbered.

(A color version of this figure is available in the online journal.)

background subtraction (using a low-order polynomial fit to each leg, with the region including the Galaxy excluded from this fit), and exclusion of the first five images in each scan leg due to boost frame transients. At 70 and $160 \mu \mathrm{m}$, the extra processing step is a pixel-dependent background subtraction for each map to remove residual detector drifts and background cirrus and zodiacal emissions. The $24 \mu \mathrm{m}$ image was saturated in the core and was therefore excluded from the photometric analysis.

Herschel/SPIRE (Pilbratt et al. 2010; Griffin et al. 2010) images at 250, 350, and $500 \mu \mathrm{m}$ were obtained on 2009 December 27 with a total integration time $4408 \mathrm{~s}$ as part of the Herschel key programme, "MESS-Mass-loss of Evolved StarS." The reduction of the SPIRE data is described in 

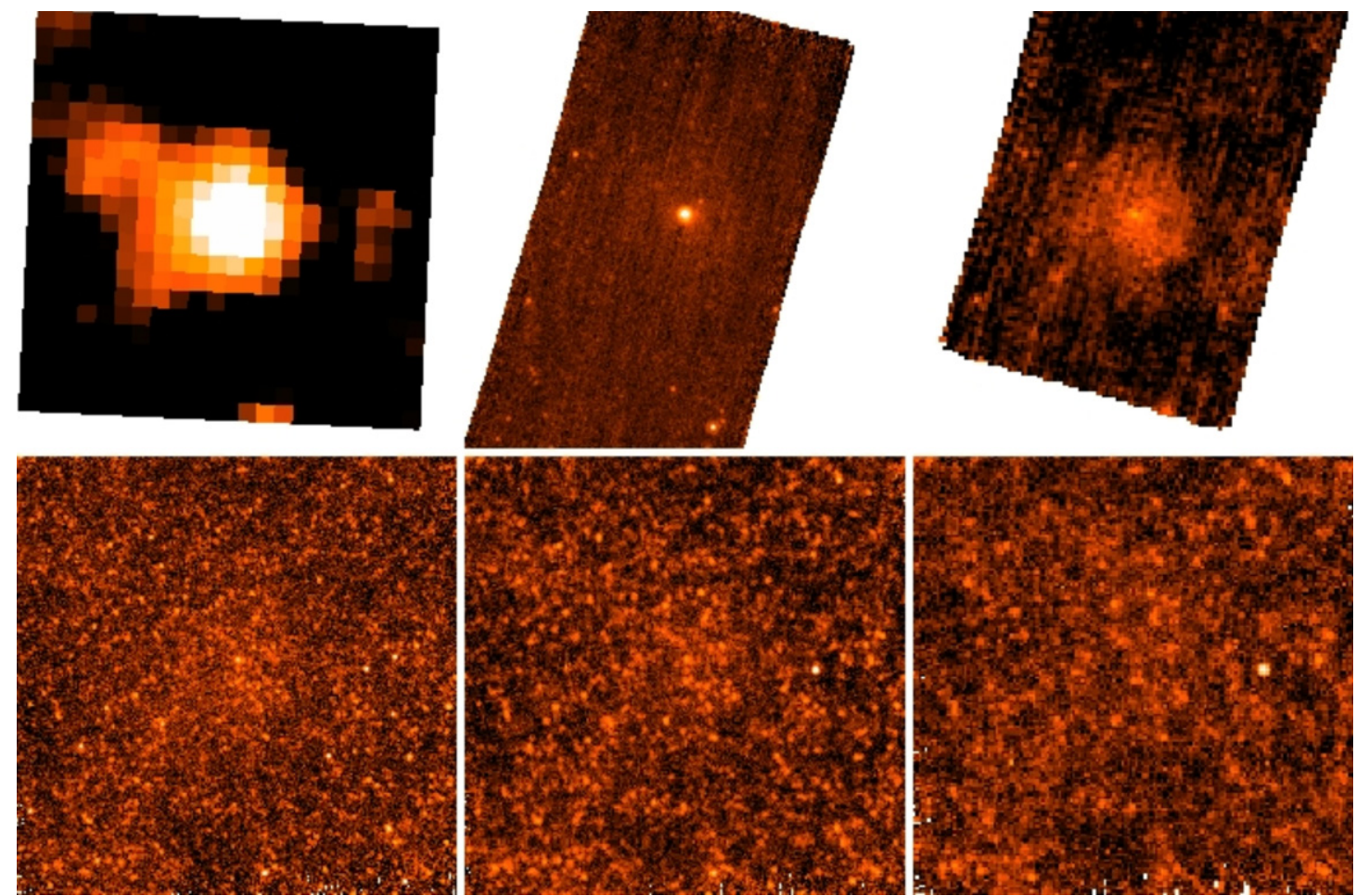

Figure 5. Far-IR images centered on $\mathrm{R} \mathrm{CrB}$ showing the large shell with a radius of $10^{\prime}$. Upper: IRAS/IRIS $100 \mu \mathrm{m}$, Spitzer/MIPS 70 and $160 \mu \mathrm{m}$. Lower: Herschel/SPIRE 250, 350, and $500 \mu \mathrm{m}$. The field is $35^{\prime} \times 35^{\prime}$.

(A color version of this figure is available in the online journal.)

Groenewegen et al. (2011). The data were processed using the standard SPIRE photometer data processing pipeline (Griffin et al. 2008, 2010; Dowell et al. 2010). The procedure for calibrating the data is described in Swinyard et al. (2010).

The IRAS imaging data have been reprocessed using a better zodiacal light subtraction and better destriping (MivilleDeschênes \& Lagache 2005). The co-added $100 \mu \mathrm{m}$ IRIS (Improved Reprocessing of the IRAS Survey) images have been downloaded for use in this study. The IRAS, Spitzer, and Herschel images are shown in Figure 5. The new images have significantly better resolution than the IRAS $100 \mu \mathrm{m}$ images. The pixel sizes are IRAS/100 (1'5), MIPS/70 (5"), MIPS/160 (16"), SPIRE/250 (6"), SPIRE/350 (10"), and SPIRE/500 (14").

\section{DISCUSSION}

$\mathrm{R}$ CrB lies at high Galactic latitude $\left(b^{\mathrm{II}}=+51^{\circ}\right)$ and so the foreground extinction is quite small, $E(B-V) \sim 0.035 \mathrm{mag}$ (Schlegel et al. 1998). At maximum light, $\mathrm{R} \mathrm{CrB} \mathrm{is} V=5.8 \mathrm{mag}$ and $B-V=0.6 \mathrm{mag}$ (Lawson et al. 1990). The absolute magnitude of $\mathrm{R} \mathrm{CrB}$ is estimated to be $M_{V}=-5$ mag based on the absolute magnitude/effective temperature relationship found for the Large Magellanic Cloud RCB stars (Alcock et al. 2001; Tisserand et al. 2009). So, for the analysis in this paper, we adopt a distance of $1.4 \mathrm{kpc}$. The most recent decline of $\mathrm{R} \mathrm{CrB}$ began in 2007 July ( JD 2454290) and the star began returning toward maximum light in the spring of 2011. Landolt \& Clem (2010) point out that the recent decline of $\mathrm{R} \mathrm{CrB}$ is among the longest ever recorded. This decline was deeper than most, bottoming out at $V \sim 15.2 \mathrm{mag}$.

\subsection{Background Objects}

As shown in Figure 1, the Gemini/GMOS and HST/WFPC2 imaging was obtained when the star was at $V \sim 15 \mathrm{mag}$ or about

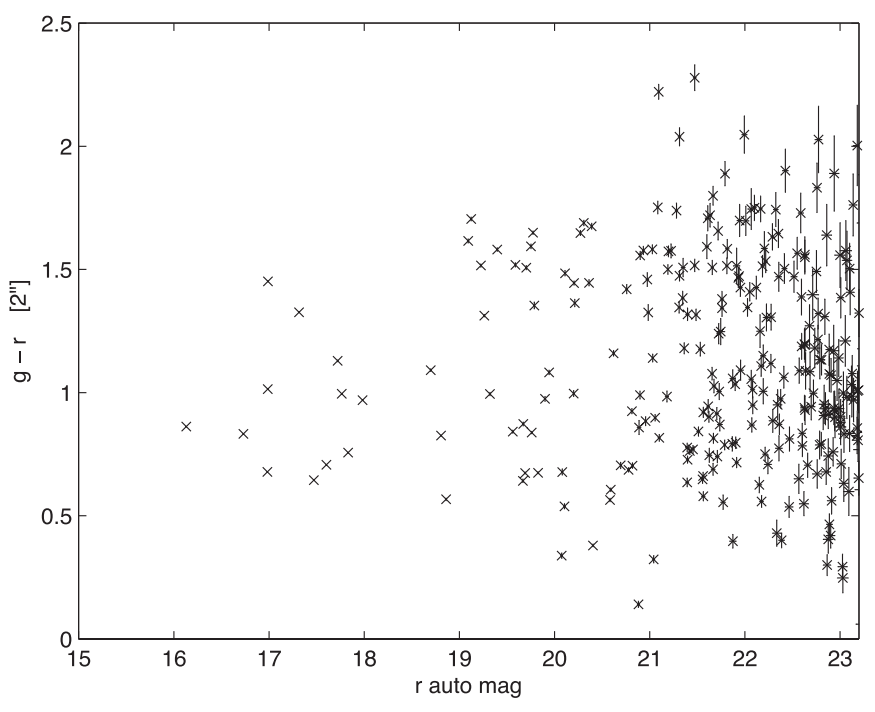

Figure 6. CMD based on the Gemini/GMOS images. There is a clear red sequence at $g-r=1.6$ which suggests a cluster at $z \sim 0.3$. The clustering at $g-r \sim 1$ is probably $\mathrm{M}$ stars and foreground galaxies.

9 mag below maximum light. The extreme faintness of the star revealed for the first time that $\mathrm{R} \mathrm{CrB} \mathrm{lies} \mathrm{in} \mathrm{front} \mathrm{of} \mathrm{a} \mathrm{cluster} \mathrm{of}$ galaxies, shown in Figure 2.

A color-magnitude diagram (CMD) was constructed using the Gemini/GMOS images. The GMOS $g$-band image was registered to the $r$-band image. Then SExtractor was used to obtain photometry in dual image mode, with the $r$-band image used for selecting objects. For the $g-r$ colors in the CMD, photometry was obtained through $2^{\prime \prime}$ diameter apertures. The GMOS $g-r$ versus $r$ diagram, shown in Figure 6, displays a fairly clear red sequence at $g-r \sim 1.6$ and $r \sim 20$ which 
corresponds to a cluster of galaxies at $z \sim 0.3$. The sizes of the galaxies in the GMOS imaging are appropriate for this redshift.

$\mathrm{R} \mathrm{CrB}$ also lies in an SDSS strip obtained in 2005 when the star was bright. SDSS did not obtain spectra of any of the galaxies in Figure 2, but it did get spectra of about 25 galaxies within $\sim 10^{\prime}$ of $\mathrm{R} \mathrm{CrB}$. Of these 14 had redshifts in the range $0.07-0.09$, and 6 had redshifts in the range $0.16-0.18$. The SDSS spectroscopy is unable to reach normal cluster galaxies at $z \sim 0.3$ and the SDSS redshifts are likely to be those of field galaxies in the foreground of the cluster.

Landolt \& Clem (2010) were the first to point out that there is a faint red star $\sim 3^{\prime \prime}$ west of $\mathrm{R} \mathrm{CrB}$. They find that the star has a magnitude of $V=20.78 \pm 0.06$ and $B-V=0.99 \pm 0.08$. It is also clearly visible in the HST and Gemini images taken during the $\mathrm{R} \mathrm{CrB}$ minimum in Figures 3 and 4. If the star is a physical companion, it would be at a projected distance of about $4000 \mathrm{AU}$ from $\mathrm{R} \mathrm{CrB}$, and its absolute magnitude would be $\sim+10$ mag. This would imply that the star is a main-sequence $\mathrm{M}$ star; however the observed $B-V$ colors are not consistent with such a star. On the other hand, if the faint companion to $\mathrm{R} \mathrm{CrB}$ is a $\mathrm{K} 2 \mathrm{~V}$ star, which is more consistent with its colors $\left(B-V=0.91\right.$ and $M_{V}=+6.4 \mathrm{mag}$; Cox 2000), then it is at a distance of $\sim 7 \mathrm{kpc}$. In short, the companion star is likely to be a background $\mathrm{K}$ dwarf. The companion star to $\mathrm{R} \mathrm{CrB}$ is not visible in the HST/WFPC2 F469N images from 1996. The WFPC2 ETC, assuming a 20th magnitude $\mathrm{K} 0$ star, estimates that the ratio of the count rates in the three filters will be F469N/F555W/F814W =0.04/5.7/6.7 electrons pixel ${ }^{-1} \mathrm{~s}^{-1}$. So it is likely that the star was too faint to be seen in the $\mathrm{F} 469 \mathrm{~N}$ images.

\subsection{Cometary Knots Close to $\mathrm{R} C r B$}

The Gemini and HST fields centered on $\mathrm{R} \mathrm{CrB}$ are shown in Figures 3 and 4. A stellar PSF has been subtracted from the position of $\mathrm{R} \mathrm{CrB}$ for each image. The Gemini and HST images were obtained only a month apart and the same structures can be seen in both. The structures are labeled in Figure 4, showing real nebulosity as well as instrumental artifacts such as diffraction spikes and charge transfer effects. The real structures appear in multiple filters in both GMOS and WFPC2 images so are unlikely to be artifacts.

$\mathrm{R} \mathrm{CrB}$ was 9 mag fainter than at maximum light during the decline. The dust lying in front of the star is extremely optically thick so at this time we are seeing essentially zero directly transmitted light. When the star itself is completely obscured, all that remains is a fairly constant scattered-light residual that is likely to be from photons that have escaped around the edge of the obscuring cloud by scattering off dust lying beside or behind the star. This scattered light may be responsible for the typical flat-bottomed declines often seen in RCB star light curves. When the stellar photosphere is obscured in deep declines, RCB stars show emission lines in their spectra. Spectra were obtained of $\mathrm{R} \mathrm{CrB}$ in 2008 when it was deep in the present decline showing emission lines including the Na I D lines and the IR Ca II triplet (Rao \& Lambert 2010). These spectra show that the emission lines account for no more than $5 \%-10 \%$ of the flux in the $V$ band. $\mathrm{R}$ CrB itself appears to be marginally resolved in the WFPC2 images. There is a diffuse, nearly circular "halo" around the star. This may be the dust near the star that is scattering the light around the obscuring cloud. Jeffers et al. (2011) also suggest the presence of this halo based on the brightness of $\mathrm{R} \mathrm{CrB}$ at the bottom of its decline. The blobs visible in Figures 3 and 4 are also seen in scattered light from R CrB.
Several of the structures around $\mathrm{R}$ CrB appear as jet-like blobs pointing radially away from the star. This could either be a lighting effect created by the central star shining through holes in the dust clouds and illuminating different portions of the circumstellar dust shell, or the blobs could be "cometary" knots. The RCB star UW Cen shows both illumination structures and cometary structures (Clayton et al. 1999; G. C. Clayton et al. 2011, in preparation). Figure 7 shows a comparison of the blobs seen in R CrB and cometary globules seen in UW Cen. In particular, the globule to the southeast of $\mathrm{R} \mathrm{CrB}$ shows the typical "tadpole" shape similar to those seen in the Helix nebula (Matsuura et al. 2009). It also shows a sharp edge on the side toward the star with the brightness fading away from the star, as shown in Figure 8. The largest knot (\#1 in Figure 7) is $\sim 1000$ AU across. This is larger than the knots in the Helix which are 100-300 AU in size with masses of $10^{-5} M_{\odot}$ (O’Dell \& Handron 1996). The simplest explanation for these cometary knots is that they are caused by a stellar wind streaming past a density enhancement moving slowly away from the star (García-Segura et al. 2006). There is evidence for both low- $\left(10-20 \mathrm{~km} \mathrm{~s}^{-1}\right)$ and high- $\left(200-400 \mathrm{~km} \mathrm{~s}^{-1}\right)$ velocity gas streaming away from RCB stars (Clayton et al. 1992, 2003; Garcia-Hernandez et al. 2011). Simulations show that when a slow wind is overtaken by a fast wind, the gas-gas interaction could create knot-like shapes (Pittard et al. 2005). Recent investigations using a hydrodynamics code including dust show that small dust grains $(\lesssim 0.045 \mu \mathrm{m})$ will follow the motion of the gas closely (van Marle et al. 2011). The UW Cen nebula, about $15^{\prime \prime}$ across, resembles a fossil PN shell where the gas is neutral and the shell is seen in light scattered from dust (Clayton et al. 1999). UW Cen is at a distance of $\sim 5.5 \mathrm{kpc}$ so if it were at the distance of $\mathrm{R} \mathrm{CrB}$, its nebula would be about $1^{\prime}$ in size. As described below, the diffuse nebulosity around $\mathrm{R} \mathrm{CrB}$ extends at least $10^{\prime}$ from the star but no blobs or density concentrations are seen more than a few arcseconds from the star.

Jeffers et al. (2011) report imaging polarimetry of the cometary knot southeast of R CrB (\#1 in Figure 7). Combining those data with the WFPC2 images presented here, they infer that the knot is at a scattering angle of $105^{\circ}$ and has a dust mass of $\sim 10^{-6} M_{\odot}$. It lies about $1^{\prime \prime}$ from $\mathrm{R} \mathrm{CrB}$ which is about $1400 \mathrm{AU}$ from the star. At velocities of 20 and $200 \mathrm{~km} \mathrm{~s}^{-1}$, the age of the blob would be 300 or 30 years, respectively.

To estimate the mass of dust present in the knots, we make the following simplifying assumptions: (1) the illuminating flux from $\mathrm{R} \mathrm{CrB}$ is unobscured (i.e., equal to the maximum values given in Table 2), (2) the dust in the knot is optically thin, such that all grains are illuminated and no grains are shadowed, and (3) that the grains can be described by the neutral polycyclic aromatic hydrocarbon (PAH) and graphitic solid properties from Li \& Draine (2001). Following Chevalier (1986), the flux scattered off one dust grain of radius $a$ at a distance $r$ from a source with flux $F(\lambda)$ is

$$
d F_{\mathrm{sca}}(\lambda, r, a)=\frac{Q_{\mathrm{sca}}(\lambda, a) \pi a^{2} \Phi(\lambda, a, \theta) F(\lambda)}{4 \pi r^{2}},
$$

where $Q_{\text {sca }}$ is the scattering efficiency, $\Phi$ is the Henyey \& Greenstein (1941) phase function, and $\theta$ is the scattering angle. If one assumes that the dust grains have uniform size, the mass of grains $\left(M_{\mathrm{gr}}\right)$ in a knot that produces a total scattered flux $\left(F_{\text {sca }}\right)$ is

$$
M_{\mathrm{gr}}=\frac{16 \pi^{2} \rho a r^{2} F_{\mathrm{sca}}}{3 F(\lambda) Q_{\mathrm{sca}}(\lambda, a) \Phi(\lambda, a, \theta)},
$$



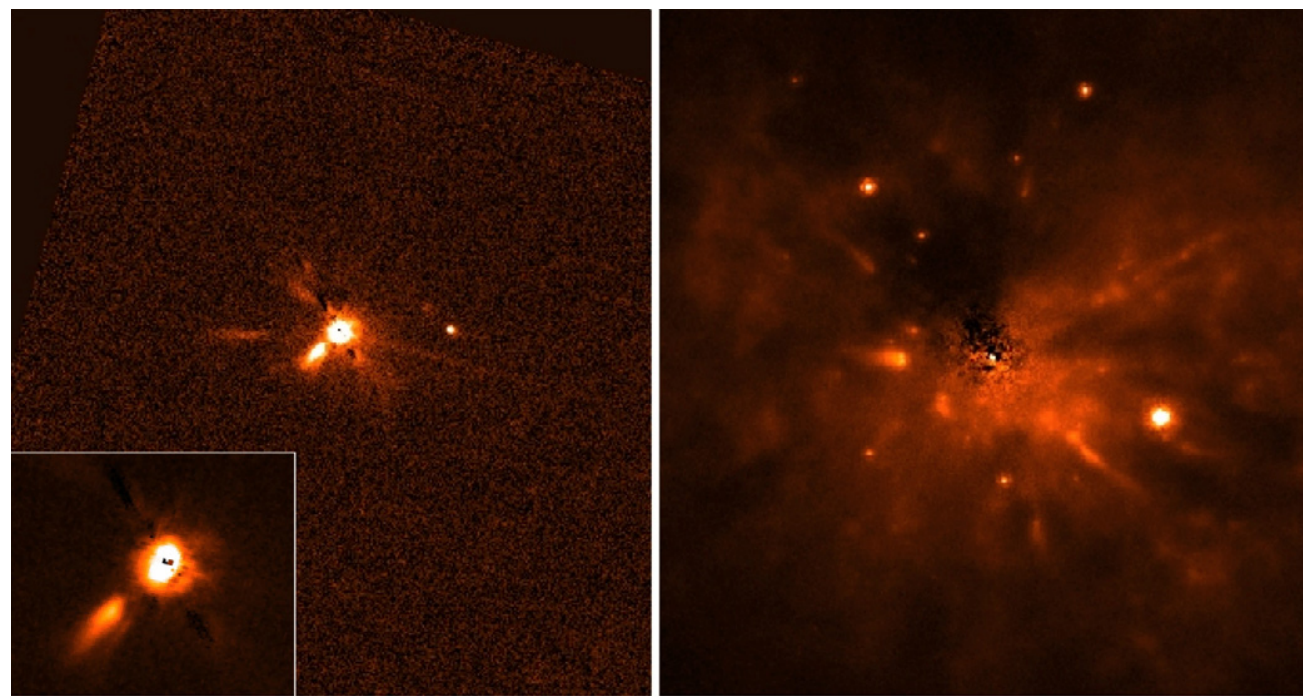

Figure 7. HST/WFPC2 F555W image $\left(17^{\prime \prime} \times 17^{\prime \prime}\right)$ of R CrB (left) and WFPC2 $606 \mathrm{~W}$ image $\left(12^{\prime \prime} \times 12^{\prime \prime}\right)$ of UW Cen (right). The inset shows the cometary globule (\#1) to the lower left of R CrB.

(A color version of this figure is available in the online journal.)

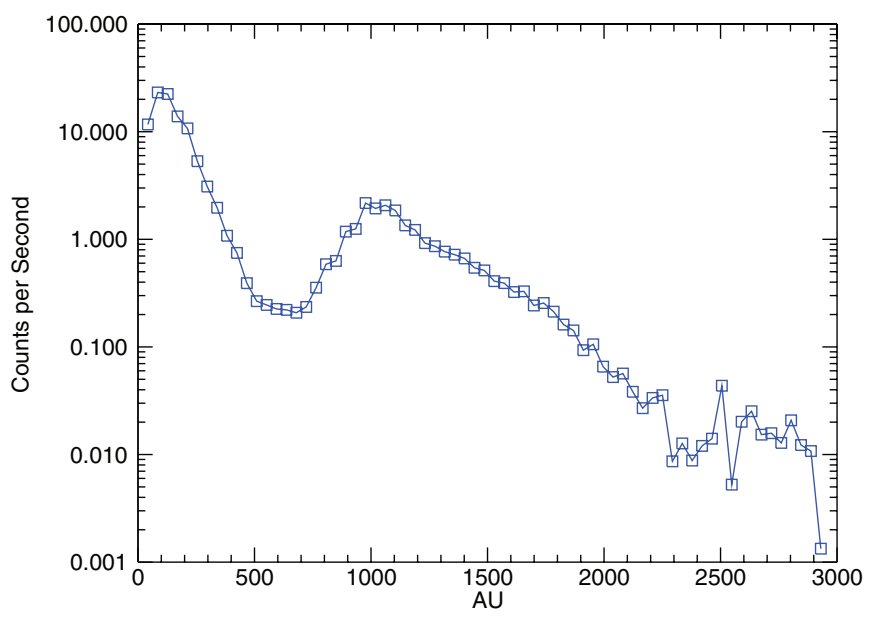

Figure 8. Cut from the center of the PSF subtracted through the large cometary knot (\#1) in the HST/WFPC2 F555W image. The cometary globule lies at 1000-2000 AU from the star.

(A color version of this figure is available in the online journal.)

where $\rho=2.24 \mathrm{~g} \mathrm{~cm}^{-3}$ is the density of carbonaceous dust. If one instead adopts an MRN size distribution (Mathis et al. 1977) such that the number density of grains $n_{\mathrm{gr}} \propto a^{-3.5}$ then

$$
M_{\mathrm{gr}}=\frac{4 r^{2} F_{\mathrm{sca}}}{F(\lambda) \int 0.224 \pi a^{-1.5} Q_{\mathrm{sca}}(\lambda, a) \Phi(\lambda, a, \theta) d a},
$$

where the numeric constant is the normalization factor from Weingartner \& Draine (2001), and assuming a gas-to-dust ratio of 100. Finally, if the dust is assumed to lie in a spherical shell of inner and outer radii $R_{\mathrm{in}}$ and $R_{\text {out }}$ then $M_{\mathrm{gr}}$ must be integrated over the volume of the shell. Since

$$
\int_{0}^{\pi} \Phi(\lambda, a, \theta) \sin \theta d \theta=2,
$$

the total grain mass for a single-sized model equals

$$
M_{\mathrm{gr}}=\frac{16 \pi \rho a F_{\mathrm{sca}}}{9 F(\lambda) Q_{\mathrm{sca}}(\lambda, a)} \frac{R_{\mathrm{out}}^{3}-R_{\mathrm{in}}^{3}}{R_{\mathrm{out}}-R_{\mathrm{in}}}
$$

Table 2

\begin{tabular}{lcc}
\multicolumn{3}{c}{ R CrB Photometry } \\
\hline \hline Band $^{\mathrm{a}}$ & $\begin{array}{c}\text { Flux } \\
(\mathrm{Jy})\end{array}$ & $\begin{array}{c}\sigma \\
(\mathrm{Jy})\end{array}$ \\
\hline$U$ & $4.53 \mathrm{e}+00$ & $3.90 \mathrm{e}-02$ \\
$B$ & $1.16 \mathrm{e}+01$ & $1.07 \mathrm{e}-01$ \\
$V$ & $1.79 \mathrm{e}+01$ & $1.66 \mathrm{e}-01$ \\
$R_{C}$ & $2.05 \mathrm{e}+01$ & $1.90 \mathrm{e}-01$ \\
$I_{C}$ & $2.07 \mathrm{e}+01$ & $1.91 \mathrm{e}-01$ \\
$J$ & $1.77 \mathrm{e}+01$ & $1.65 \mathrm{e}-01$ \\
$H$ & $1.43 \mathrm{e}+01$ & $1.00 \mathrm{e}-01$ \\
$K$ & $1.43 \mathrm{e}+01$ & $1.33 \mathrm{e}-01$ \\
$L$ & $2.56 \mathrm{e}+01$ & $2.36 \mathrm{e}-01$ \\
$A K A R I / 9$ & $5.30 \mathrm{e}+01$ & $2.44 \mathrm{e}+00$ \\
$A K A R I / 18$ & $2.15 \mathrm{e}+01$ & $2.90 \mathrm{e}-02$ \\
$I R A S / 12$ & $3.89 \mathrm{e}+01$ & $1.55 \mathrm{e}+00$ \\
$I R A S / 25$ & $1.71 \mathrm{e}+01$ & $6.84 \mathrm{e}-01$ \\
$I R A S / 60$ & $3.94 \mathrm{e}+00$ & $3.15 \mathrm{e}-01$ \\
MIPS/70 & $2.03 \mathrm{e}+00$ & $3.4 \mathrm{E}-02$ \\
$A K A R I / 90$ & $1.49 \mathrm{e}+00$ & $1.14 \mathrm{e}-01$ \\
$I R A S / 100$ & $2.00 \mathrm{e}+00$ & $1.60 \mathrm{e}-01$ \\
MIPS160 & $2.97 \mathrm{E}-01$ & $9.36 \mathrm{E}-03$ \\
SPIRE/250 & $7.81 \mathrm{E}-02$ & $1.17 \mathrm{E}-02$ \\
SPIRE/350 & $3.40 \mathrm{E}-02$ & $5.10 \mathrm{E}-03$ \\
SPIRE/500 & $1.25 \mathrm{E}-02$ & $4.34 \mathrm{E}-03$ \\
\hline
\end{tabular}

Note. ${ }^{\text {a }}$ Maximum light $U B V R I$ photometry from Cottrell et al. (1990). JHKL photometry from Feast et al. (1997).

Of the knots identified in Figure 4, numbers 4 and 6 are coincident with diffraction spikes, and we refrain from estimating their dust masses. For the others, the total flux from each knot was measured through manually sized elliptical apertures. These values are reported in Table 3. For each knot, models with single grain sizes of $0.001,0.01,0.1$, and $1.0 \mu \mathrm{m}$, as well as full MRN distributions, were tested for scattering angles from $10^{\circ}$ to $170^{\circ}$ (in increments of $10^{\circ}$ ) to find which combinations of grain sizes and scattering angles yielded equivalent dust masses for both the F555W and F814W photometry. The best-fitting models, including the grain sizes, scattering angle, resulting clump distance from $\mathrm{R} \mathrm{CrB}$, and estimated dust mass, are also listed in Table 3. The model dust masses are $10^{-10}$ to $10^{-7} M_{\odot}$. Note that 
Table 3

Cometary Knot Models

\begin{tabular}{|c|c|c|c|c|c|c|}
\hline Knot & $\begin{array}{l}F_{\mathrm{F} 555 \mathrm{~W}} \\
\left(10^{-18} \mathrm{erg} \mathrm{cm}^{-2} \mathrm{~s}\right.\end{array}$ & $\begin{array}{r}F_{\mathrm{F} 814 \mathrm{~W}} \\
\left.\mathrm{~s}^{-1} \AA^{-1}\right)\end{array}$ & $\begin{array}{l}\text { Grain } \\
\text { Model }\end{array}$ & $\begin{array}{c}\theta \\
\left({ }^{\circ}\right)\end{array}$ & $\begin{array}{c}r \\
(\mathrm{AU})\end{array}$ & $\begin{array}{c}M_{\mathrm{gr}} \\
\left(M_{\odot}\right)\end{array}$ \\
\hline \multirow[t]{2}{*}{1} & 723 & 261 & $0.1 \mu \mathrm{m}$ & 110 & 1500 & $3.7 \times 10^{-9}$ \\
\hline & & & MRN & 35 & 2500 & $1.6 \times 10^{-8}$ \\
\hline 2 & 45 & 12 & $0.1 \mu \mathrm{m}$ & 70 & 4700 & $1.2 \times 10^{-9}$ \\
\hline \multirow[t]{3}{*}{3} & 32 & 4 & $0.01 \mu \mathrm{m}$ & Varies & $\mathrm{N} / \mathrm{A}$ & $(1-3) \times 10^{-7}$ \\
\hline & & & $0.001 \mu \mathrm{m}$ & Varies & $\mathrm{N} / \mathrm{A}$ & $(1-5) \times 10^{-4}$ \\
\hline & & 6 & $0.1 \mu \mathrm{m}$ & 50 & 3000 & $2.5 \times 10^{-10}$ \\
\hline Halo & 15600 & 10600 & $1.0 \mu \mathrm{m}$ & $\mathrm{N} / \mathrm{A}$ & $\lesssim 400$ & $(2-4) \times 10^{-8}$ \\
\hline
\end{tabular}

if a model is not listed, it failed to reproduce the photometry in both filters.

Knot 3 has very low flux in the F814W filter, resulting in a large uncertainty with the flux between 4 and $6 \times$ $10^{-18} \mathrm{erg} \mathrm{cm} \mathrm{cm}^{-2} \mathrm{~s}^{-1} \AA^{-1}$. For this reason, results are shown for the upper and lower flux range. Since small grains scatter isotropically, there were no preferable scattering angles for this knot using the smaller single grain-size models, making a distance estimate from R CrB impossible. Owing to the very bright F814W flux from knot 5, we find there is no dust-scattering model that adequately reproduces the fluxes in both filters.

To estimate the dust mass producing the halo (i.e., the slightly resolved profile around $\mathrm{R} \mathrm{CrB}$ ), we assume that all of its flux is light scattered by a thin circumstellar shell. We find that a limb-brightened shell of optically thin dust can be up to 0.3 in radius and still produce a profile consistent with that of $\mathrm{R} \mathrm{CrB}$ in the 2009 HST images. The flux of the halo, as well as the bestfitting dust model that produces this scattered light, is listed at the bottom of Table 3 .

Note that our first modeling assumption implies that the masses derived above are lower limits, since more dust is required if the incident flux is lower than that of $\mathrm{R} \mathrm{CrB}$ at maximum light. As a quick check of the second assumption, we assume that the knots are ellipsoids with the same axes as those used to measure the fluxes. In knot 1 , there are $8 \times 10^{38}$ grains of radius $0.1 \mu \mathrm{m}$, corresponding to a number density of $n_{\mathrm{gr}}=$ $8 \times 10^{-10} \mathrm{~cm}^{-3}$. The optical depth $\tau=n_{\mathrm{gr}} L\left(Q_{\mathrm{sca}}+Q_{\mathrm{abs}}\right) \pi a^{2}$, where $L$ is the line-of-sight distance through the dust and $Q_{\text {abs }}$ is the absorption efficiency. The proposed ellipsoid has a depth around $10^{16} \mathrm{~cm}$, yielding an approximate $V$-band optical depth of $8 \times 10^{-3}$, which is sufficiently thin to warrant the assumption that all grains are illuminated by the star. See Section 3.3 for a justification of the last assumption, namely, that the dust is purely carbonaceous.

\subsection{The Large Far-IR Dust Shell}

$\mathrm{R} \mathrm{CrB}$ has an IRAS shell with a radius of $10^{\prime}, 4 \mathrm{pc}$ at a distance of $1.4 \mathrm{kpc}$ (Gillett et al. 1986). The new MIPS images at 70 and $160 \mu \mathrm{m}$, shown in Figure 5 along with the IRAS $100 \mu \mathrm{m}$ image, confirm the presence of a large dust shell. It is also visible in the SPIRE images at 250 and $350 \mu \mathrm{m}$. It is not clearly visible at $500 \mu \mathrm{m}$. The new MIPS and SPIRE images have significantly better resolution than IRAS. These new images imply that the shell is spherical in shape and that the component to the northeast of the nebula in the IRAS $100 \mu \mathrm{m}$ image is detached from the R CrB shell in the MIPS and SPIRE images. The SPIRE images, in particular, are full of point sources, which are background galaxies. To determine how much of the nebulosity seen in the IRAS, MIPS, and SPIRE images might be due to blended emission from background galaxies, we used SExtractor to identify and remove point sources from the SPIRE $250 \mu \mathrm{m}$ image. The result is shown in Figure 9. This clearly shows that the dust nebulosity around $\mathrm{R} \mathrm{CrB}$ is not due to a concentration of galaxies in a cluster behind the star. There is significant diffuse far-IR emission, centered on $\mathrm{R} \mathrm{CrB}$. To get a clearer idea of the extent of the nebulosity at different IR wavelengths, the average brightness in $1^{\prime}$ concentric rings from $\mathrm{R}$ CrB was calculated and plotted in Figure 10. These new data show that the shell is possibly even larger than that seen by IRAS. The MIPS $70 \mu \mathrm{m}$ image also has a central elongated structure running almost east-west that has a diameter of $\sim 3^{\prime}$.

At the assumed distance of $\mathrm{R} \mathrm{CrB}$, the very extended IR shell extends about $8 \times 10^{5} \mathrm{AU}$ from the star. The dust mass and total mass of this shell have previously been estimated to be $\sim 10^{-3}$ to $10^{-2} M_{\odot}$ and $\sim 0.3-3 M_{\odot}$, respectively (Gillett et al. 1986). Gillett et al. estimate that the dust temperature in the shell is 25-30 K. Rao \& Nandy (1986) find a hot component close to the star with a dust mass of $5 \times 10^{-7} M_{\odot}$ and a cool component with a dust mass of $10^{-2}$ to $10^{-3} M_{\odot}$.

Photometry available from the literature for $\mathrm{R} \mathrm{CrB}$ has been combined with new photometry using the MIPS and SPIRE images. The data, summarized in Table 2 and plotted in Figure 11, consist of visible photometry at maximum light, $J H K L$, as well as IRAS photometry (Walker 1985; Rao \& Nandy 1986). AKARI/IRC and FIS (Infrared Camera and FarInfrared Surveyor, respectively) photometry are also available (Murakami et al. 2007; Ishihara et al. 2010). Fluxes were extracted from SPIRE $(250,350,500 \mu \mathrm{m})$ using Starfinder (Diolaiti et al. 2000). Starfinder produced PSF fitted photometry using PSFs which were downloaded from the Herschel Science Center. No aperture corrections were applied since the PSFs used were of sufficiently large diameter. Aperture photometry was also performed as a test of the Starfinder results. The photometric uncertainties quoted in Table 2 for SPIRE are $15 \%$, or the Starfinder uncertainties plus calibration errors of $5 \%$ whichever is higher (Swinyard et al. 2010).

We have modeled the spectral energy distribution (SED) of $\mathrm{R}$ CrB using a Monte Carlo radiative transfer code which includes nonisotropic scattering, polarization, and thermal emission from dust in a spherical-polar grid (Whitney et al. 2003a, 2003b; Robitaille et al. 2006). All of the models were done with amorphous carbon dust and an MRN size distribution (Mathis et al. 1977). We have assumed a mass gas-to-dust ratio of 100 . The best fit to the R CrB SED is shown in Figure 11. A good fit was obtained to both the hotter dust centered on the star, as well as the cooler, large diffuse nebula. Heating by the interstellar radiation field is included. The best-fitting model is a small disk surrounded by a large diffuse envelope. The disk has inner and outer radii of 42 and $150 \mathrm{AU}$, and the envelope has inner 

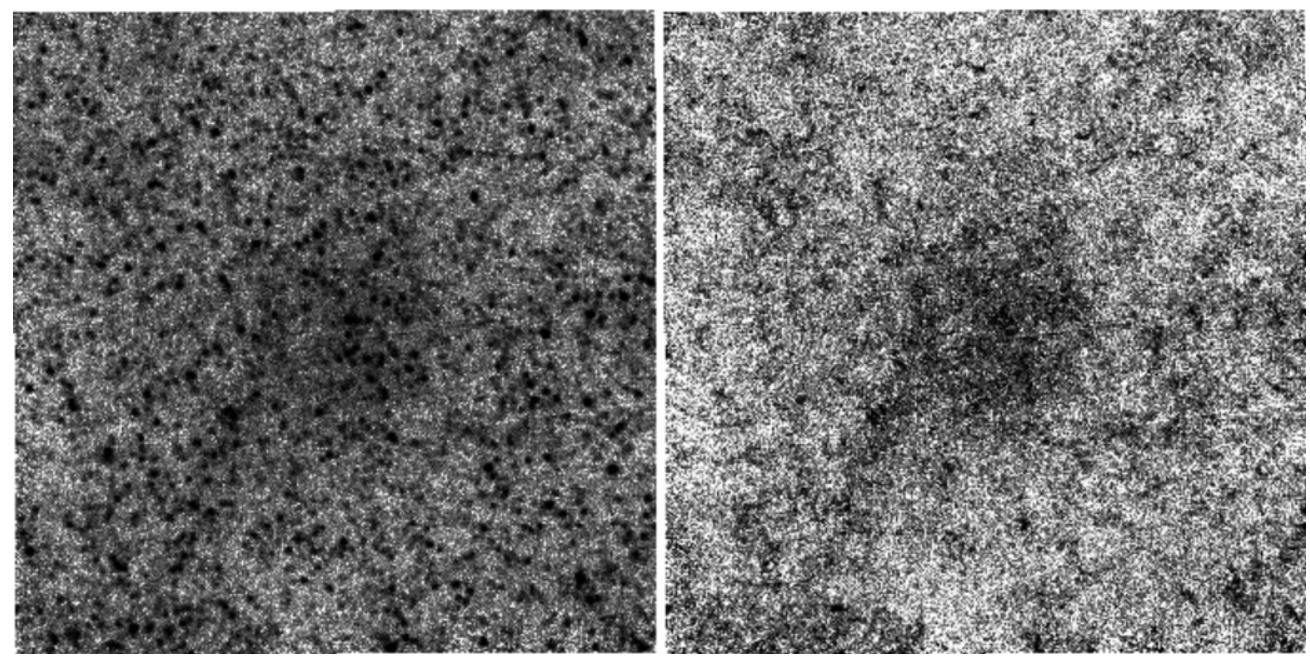

Figure 9. Left: Herschel/SPIRE $250 \mu \mathrm{m}$ image centered on R CrB. Right: same image with point sources subtracted to show diffuse IR emission is present. The field is $34^{\prime} \times 34^{\prime}$.

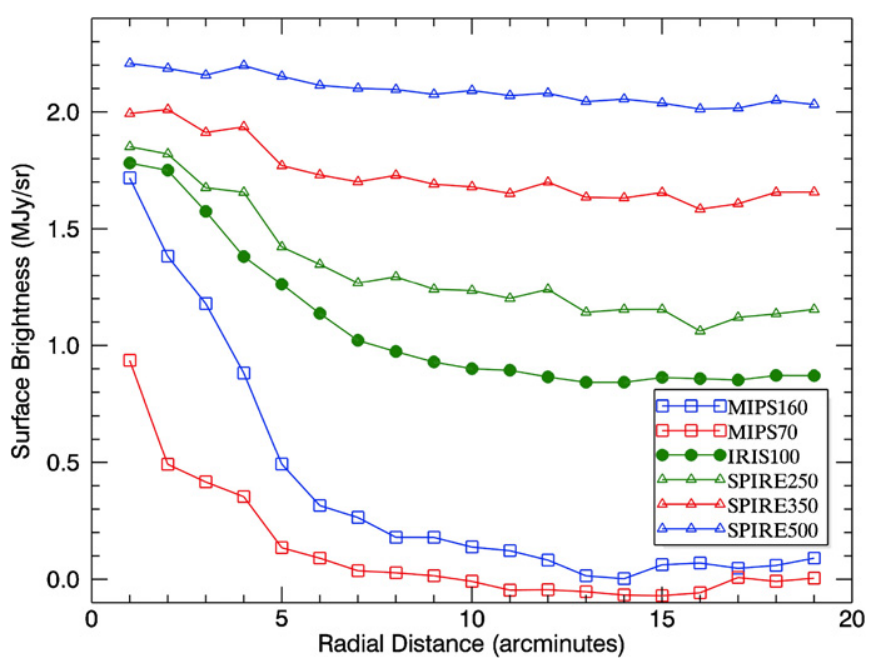

Figure 10. Surface brightness of diffuse emission surrounding $\mathrm{R} \mathrm{CrB}$ in one arcminute rings. The curves have been shifted vertically for clarity.

(A color version of this figure is available in the online journal.)

and outer radii of 1.3 and $8.8 \times 10^{5} \mathrm{AU}$. The envelope has a bipolar cavity carved out with an opening angle of $30^{\circ}$. The $A_{V}$ through the envelope is quite low, less than 0.01 mag over a wide range of viewing angles $\left(0^{\circ}-75^{\circ}\right)$. The disk dust mass is $3.5 \times 10^{-6} M_{\odot}$ and a total mass of $3.5 \times 10^{-4} M_{\odot}$. Assuming a density profile of $r^{-2}$ which is appropriate for dust accelerated away from the star radially by radiation pressure, the dust mass of the outer envelope is $2 \times 10^{-2} M_{\odot}$ and the total mass (dust + gas) is $2 M_{\odot}$. The gas may be dragged along with the dust or it may become decoupled.

\subsection{The Evolution of $R \mathrm{CrB}$ and Its Dust}

The generally accepted model for dust formation in the RCB stars, the condensation of carbon dust along the line of sight, was suggested over 70 years ago (Loreta 1935; O'Keefe 1939; Clayton 1996). The evolution of RCB star spectra and light curves during declines is consistent with dust that forms close to the stellar atmosphere and then is accelerated to hundreds of $\mathrm{km} \mathrm{s}^{-1}$ by radiation pressure (Clayton et al. 1992; Whitney et al. 1993). If the dust forms near the star, it will accelerate very quickly, possibly reaching $200 \mathrm{~km} \mathrm{~s}^{-1}$ in 30 days. Other

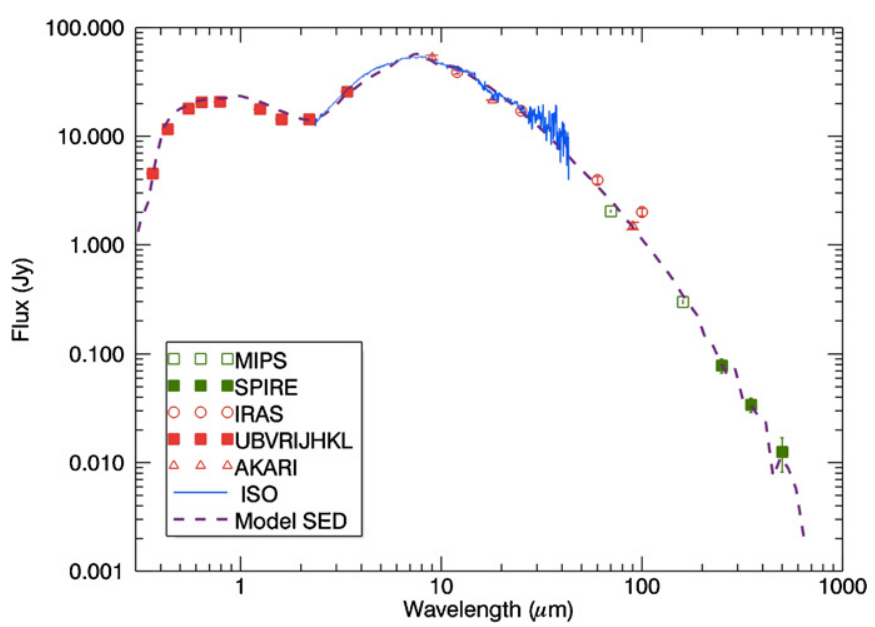

Figure 11. Maximum-light SED for R CrB. Filled red squares: UBVRIJHKLM; open red circles: IRAS $(12,25,60,100 \mu \mathrm{m})$; open green squares: MIPS (70, $160 \mu \mathrm{m})$; filled green squares: SPIRE $(250,350,500 \mu \mathrm{m})$; open red triangles: AKARI $(9,18,90 \mu \mathrm{m})$; blue line: Infrared Space Observatory spectrum. The dust associated with $\mathrm{R} \mathrm{CrB}$ was clearly detected in all the SPIRE bands. Also plotted is the best-fitting radiative transfer model to the SED (purple dashed line).

(A color version of this figure is available in the online journal.)

evidence supports a much slower expansion (e.g., GarciaHernandez et al. 2011, and references therein). The observed timescales for RCB dust formation fit in well with those calculated by carbon chemistry models (Feast 1986; Woitke et al. 1996; Rao \& Lambert 2010).

The dust forming around $\mathrm{R} \mathrm{CrB}$ is not in a complete shell but rather in small "puffs" (Clayton 1996). When a puff forms along the line of sight to the star we see a decline. Estimates of the covering factor of the clouds around RCB stars during declines, from both extinction studies and IR re-emission of stellar radiation, indicate that $f<0.5$ (Feast et al. 1997; Clayton et al. 1999; Hecht et al. 1984, 1998). The typical puff causing a decline is thought to have a dust mass of $\sim 10^{-8} M_{\odot}$ (Feast 1986; Clayton et al. 1992). There is strong evidence that the puffs form in or near the surface of the RCB star due to density and temperature perturbations caused by stellar pulsations (Woitke et al. 1996; Crause et al. 2007). The RCB stars show regular or semi-regular pulsation periods in the 40-100 day range (Lawson 
et al. 1990). R CrB itself does not have one regular period but has shown periods of 40 and 51 days (Fernie \& Lawson 1993).

For this recent deep decline, with $A_{V}=9 \mathrm{mag}$, assuming the dust forms at $2 R_{\star}\left(R_{\star}=85 R_{\odot}\right)$, and a puff subtends a fractional solid angle of 0.05 , then the dust mass will be $\sim 10^{-8} M_{\odot}$. The puff would be accelerated quickly by radiation pressure and would dissipate rapidly so dust must be formed continually by $\mathrm{R} \mathrm{CrB}$ to maintain itself in a deep decline for 4 years. If a puff is formed during each pulsation period of $\sim 40$ days, $\mathrm{R} \mathrm{CrB}$ would be forming about $10^{-7} M_{\odot}$ of dust per year. Assuming a gas-to-dust ratio of 100 (Whittet 2003), the total mass loss per year is $10^{-5} M_{\odot}$. The estimated masses of the puffs causing the declines and the cometary knots are similar. The puffs cause declines when they form directly in our line of sight and may be seen as cometary knots when they form to the side of or behind $\mathrm{R}$ CrB.

Little is known about the lifetime of the RCB phase. We have a lower limit from the fact that $\mathrm{R} \mathrm{CrB}$ itself was discovered to have large brightness variations 200 years ago (Pigott \& Englefield 1797). Assuming a velocity of $20 \mathrm{~km} \mathrm{~s}^{-1}$ then the large diffuse dust shell around $\mathrm{R} \mathrm{CrB}$ would take $10^{5} \mathrm{yr}$ to form. If the mass loss was more like the high-velocity winds seen in $\mathrm{R}$ CrB today $\left(\sim 200 \mathrm{~km} \mathrm{~s}^{-1}\right)$ then the shell would be about an order of magnitude younger (Clayton et al. 2003). If $\mathrm{R} \mathrm{CrB}$ is the result of a final-helium-shell flash rather than a white dwarf merger, then the size and timescales would be consistent with the nebulosity, now seen in far-IR emission, being a fossil PN shell. The nebulosity including cometary knots, seen around R CrB and UW Cen, shown in Figure 7, looks very much like a PN shell (Clayton et al. 1999; G. C. Clayton et al. 2011, in preparation).

$\mathrm{PNe}$ are a very late phase in the evolution of low- and intermediate-mass $\left(1-8 M_{\odot}\right)$ stars. The PN NGC 6302 is estimated to have an envelope with a total mass of $4.7 M_{\odot}$ and a central star with a progenitor mass of $5.5 M_{\odot}$ (Wright et al. 2011). So the estimate made here for the total envelope mass of $\mathrm{R} \mathrm{CrB}$ is consistent with it being a fossil PN shell. Any gas lost during the white dwarf merger would have far less mass. If the shell is an old PN shell then this would suggest that $\mathrm{R} \mathrm{CrB}$ is the product of a final-flash event rather than a white dwarf merger. The estimated mass of the envelope is consistent with a mass-loss rate of $10^{-5} M_{\odot}$ for about $10^{5} \mathrm{yr}$.

Five Galactic RCB stars, including $\mathrm{R}$ CrB, itself, show significant lithium in their atmospheres (Rao \& Lambert 1996; Asplund et al. 2000; Kipper \& Klochkova 2006). Renzini (1990) suggested that, in a final flash, the ingestion of the H-rich envelope leads to Li production through the Cameron-Fowler mechanism (Cameron \& Fowler 1971). The abundance of $\mathrm{Li}$ in the atmosphere of the final-flash star, Sakurai's object, was actually observed to increase with time (Asplund et al. 1999). In a white dwarf merger, the temperatures required for the production of ${ }^{18} \mathrm{O}$ should destroy any $\mathrm{Li}$ present. So, the simultaneous enrichment of $\mathrm{Li}$ and ${ }^{18} \mathrm{O}$ is not expected in the white dwarf merger scenario. The abundance of ${ }^{18} \mathrm{O}$ cannot be directly measured in $\mathrm{R} \mathrm{CrB}$, but it is overabundant in fluorine, which does imply a high ${ }^{18} \mathrm{O}$ abundance (Pandey et al. 2008).

About $10 \%$ of single stars will undergo a final-flash event (Iben et al. 1996). About this percentage of RCB stars (R CrB, RY Sgr, V CrA, and UW Cen) show evidence of resolved fossil dust shells (Walker 1994). R CrB is thought to be $\sim 0.8-0.9 M_{\odot}$ from pulsation modeling (Saio 2008), and this mass agrees well with the predicted mass of the merger products of a $\mathrm{CO}$ and an He white dwarf (Han 1998). On the other hand, final-flash stars, since they are single white dwarfs, should typically have masses of 0.55-0.6 $M_{\odot}$ (Bergeron et al. 2007).

\section{SUMMARY}

New images were obtained of R CrB with Gemini/GMOS, HST/WFPC2, Spitzer/MIPS, and Herschel/SPIRE just before or during its recent very long and very deep decline. At visible wavelengths with Gemini and $H S T$, the dust obscuring $\mathrm{R} \mathrm{CrB}$ acts as a natural coronagraph, allowing material close to the star to be imaged for the first time. In particular, several cometary knots have been detected within a few thousand AU of the star. The puffs of dust that cause declines in R CrB and the cometary knots have similar inferred masses so they may be the same dust clouds seen in and out of the direct line of sight to the star. Also, the existence of a cluster of galaxies directly behind $\mathrm{R} \mathrm{CrB}$ was revealed for the first time. The IR images confirm the existence of a very large diffuse shell (radius $\sim 4 \mathrm{pc}$ ) around $\mathrm{R} \mathrm{CrB}$ that contains cool dust $(\sim 25 \mathrm{~K})$ which is detected out to $500 \mu \mathrm{m}$ and has a total mass of $\sim 2 M_{\odot}$. This places a possible upper limit on the lifetime of $\mathrm{R} \mathrm{CrB}$ as an RCB of $\sim 10^{5} \mathrm{yr}$. In addition, there is no significant mass of cold dust around $\mathrm{R} \mathrm{CrB}$ that was missed by IRAS.

RCB stars are rare so they do not contribute significantly to the interstellar medium, but their continual dust formation makes them ideal laboratories for understanding dust formation in evolved stars. In particular, both the RCB stars and Type Ia supernovae may be the product of a white dwarf merger (Wheeler 1978; Webbink 1984; Clayton et al. 2007; Kilic et al. 2010). So studies of the circumstellar environments of RCB stars may provide information on the material around Type Ia supernovae. It is possible that there are two avenues for producing $\mathrm{RCB}$ stars and that $\mathrm{R} \mathrm{CrB}$ is a product of the final flash rather than the white dwarf merger. But the evidence is mixed. The IR shell and the presence of lithium point toward a possible final flash, while the relatively high mass of $\mathrm{R} \mathrm{CrB}$ and the presence of fluorine point to a white dwarf merger.

We appreciate the granting of Gemini and HST Director's Discretionary time for this project. This work is based in part on observations made with the Spitzer Space Telescope, which is operated by the Jet Propulsion Laboratory, California Institute of Technology under a contract with NASA. Support for this work was provided by NASA through an award (RSA No. 1287678) issued by JPL/Caltech. Support for Program number HST-GO-12000.01-A was provided by NASA through a grant from the Space Telescope Science Institute, which is operated by the Association of Universities for Research in Astronomy, Incorporated, under NASA contract NAS5-26555. A portion of these data was obtained at the Gemini Observatory, which is operated by the Association of Universities for Research in Astronomy (AURA) under a cooperative agreement with the NSF on behalf of the Gemini partnership. Herschel is an ESA space observatory with science instruments provided by European-led Principal Investigator consortia and with important participation from NASA. Funding for the SDSS and SDSS-II has been provided by the Alfred P. Sloan Foundation, the Participating Institutions, the National Science Foundation, the U.S. Department of Energy, the National Aeronautics and Space Administration, the Japanese Monbukagakusho, the Max Planck Society, and the Higher Education Funding Council for England. We acknowledge with thanks the variable star observations from the AAVSO International Database contributed by observers worldwide and used in this research. 


\section{REFERENCES}

Alcock, C., Allsman, R. A., Alves, D. R., et al. 2001, ApJ, 554, 298

Asplund, M., Gustafsson, B., Lambert, D. L., \& Rao, N. K. 2000, A\&A, 353, 287

Asplund, M., Lambert, D. L., Kipper, T., Pollacco, D., \& Shetrone, M. D. 1999, A\&A, 343, 507

Bergeron, P., Gianninas, A., \& Boudreault, S. 2007, in ASP Conf. Ser. 372, 15th European Workshop on White Dwarfs, ed. R. Napiwotzki \& M. R. Burleigh (San Francisco, CA: ASP), 29

Bright, S. N., Chesneau, O., Clayton, G. C., et al. 2011, MNRAS, 414, 1195

Cameron, A. G. W., \& Fowler, W. A. 1971, ApJ, 164, 111

Chevalier, R. A. 1986, ApJ, 308, 225

Clayton, G. C. 1996, PASP, 108, 225

Clayton, G. C., \& Ayres, T. R. 2001, ApJ, 560, 986

Clayton, G. C., Bjorkman, K. S., Nordsieck, K. H., Zellner, N. E. B., \& SchulteLadbeck, R. E. 1997, ApJ, 476, 870

Clayton, G. C., Geballe, T. R., \& Bianchi, L. 2003, ApJ, 595, 412

Clayton, G. C., Geballe, T. R., Herwig, F., Fryer, C., \& Asplund, M. 2007, ApJ, 662,1220

Clayton, G. C., Herwig, F., Geballe, T. R., et al. 2005, ApJ, 623, L141

Clayton, G. C., Kerber, F., Gordon, K. D., et al. 1999, ApJ, 517, L143

Clayton, G. C., Whitney, B. A., Stanford, S. A., \& Drilling, J. S. 1992, ApJ, 397,652

Cottrell, P. L., Lawson, W. A., \& Buchhorn, M. 1990, MNRAS, 244, 149

Cox, A. N. 2000, in Allen's Astrophysical Quantities, ed. A. N. Cox (New York, NY: AIP Press; Springer)

Crause, L. A., Lawson, W. A., \& Henden, A. A. 2007, MNRAS, 375, 301

de Laverny, P., \& Mékarnia, D. 2004, A\&A, 428, L13

Diolaiti, E., Bendinelli, O., Bonaccini, D., et al. 2000, A\&AS, 147, 335

Dowell, C. D., Pohlen, M., Pearson, C., et al. 2010, Proc. SPIE, 7731, 773136

Feast, M. W. 1986, in IAU Colloq. 87, Hydrogen Deficient Stars and Related Objects, ed. K. Hunger, D. Schoenberner, \& N. Kameswara Rao (Astrophysics and Space Science Library, Vol. 128; Dordrecht: Reidel), 151

Feast, M. W., Carter, B. S., Roberts, G., Marang, F., \& Catchpole, R. M. 1997, MNRAS, 285, 317

Fernie, J. D., \& Lawson, W. A. 1993, MNRAS, 265, 899

Garcia-Hernandez, D. A., Kameswara Rao, N., \& Lambert, D. L. 2011, arXiv: 1107.1185

García-Hernández, D. A., Lambert, D. L., Kameswara Rao, N., Hinkle, K. H., \& Eriksson, K. 2010, ApJ, 714, 144

García-Segura, G., López, J. A., Steffen, W., Meaburn, J., \& Manchado, A. 2006, ApJ, 646, L61

Gillett, F. C., Backman, D. E., Beichman, C., \& Neugebauer, G. 1986, ApJ, 310, 842

Gordon, K. D., Rieke, G. H., Engelbracht, C. W., et al. 2005, PASP, 117, 503

Griffin, M. J., Abergel, A., Abreu, A., et al. 2010, A\&A, 518, L3

Griffin, M., Swinyard, B., Vigroux, L., et al. 2008, Proc. SPIE, 7010, 701006

Groenewegen, M. A. T., Waelkens, C., Barlow, M. J., et al. 2011, A\&A, 526, 162

Han, Z. 1998, MNRAS, 296, 1019

Hecht, J. H., Clayton, G. C., Drilling, J. S., \& Jeffery, C. S. 1998, ApJ, 501, 813

Hecht, J. H., Holm, A. V., Donn, B., \& Wu, C.-C. 1984, ApJ, 280, 228

Henyey, L. G., \& Greenstein, J. L. 1941, ApJ, 93, 70

Iben, I., Jr., Tutukov, A. V., \& Yungelson, L. R. 1996, ApJ, 456, 750

Ishihara, D., Onaka, T., Kataza, H., et al. 2010, A\&A, 514, A1

Jeffers, S. V., et al. 2011, A\&A, submitted

Kilic, M., Brown, W. R., Allende Prieto, C., Kenyon, S. J., \& Panei, J. A. 2010, ApJ, 716, 122
Kipper, T., \& Klochkova, V. G. 2006, Balt. Astron., 15, 531

Landolt, A. U., \& Clem, J. L. 2010, PASP, 122, 541

Lawson, W. A., Cottrell, P. L., Kilmartin, P. M., \& Gilmore, A. C. 1990, MNRAS, 247, 91

Leão, I. C., de Laverny, P., Chesneau, O., Mékarnia, D., \& de Medeiros, J. R. 2007, A\&A, 466, L1

Li, A., \& Draine, B. T. 2001, ApJ, 554, 778

Loreta, E. 1935, Astron. Nachr., 254, 151

Mathis, J. S., Rumpl, W., \& Nordsieck, K. H. 1977, ApJ, 217, 425

Matsuura, M., Speck, A. K., McHunu, B. M., et al. 2009, ApJ, 700, 1067

Miville-Deschênes, M., \& Lagache, G. 2005, ApJS, 157, 302

Murakami, H., Baba, H., Barthel, P., et al. 2007, PASJ, 59, 369

O’Dell, C. R., \& Handron, K. D. 1996, AJ, 111, 1630

Ohnaka, K., Beckmann, U., Berger, J.-P., et al. 2003, A\&A, 408, 553

O'Keefe, J. A. 1939, ApJ, 90, 294

Pandey, G., Lambert, D. L., \& Rao, N. K. 2008, ApJ, 674, 1068

Pigott, E., \& Englefield, H. C. 1797, R. Soc. of Lond. Phil. Trans. I, 87, 133

Pilbratt, G. L., Riedinger, J. R., Passvogel, T., et al. 2010, A\&A, 518, L1

Pittard, J. M., Dyson, J. E., Falle, S. A. E. G., \& Hartquist, T. W. 2005, MNRAS, 361, 1077

Pollacco, D. L., Hill, P. W., Houziaux, L., \& Manfroid, J. 1991, MNRAS, 248, 1P

Rao, N. K., \& Lambert, D. L. 1996, in ASP Conf. Ser. 96, Hydrogen Deficient Stars, ed. C. S. Jeffery \& U. Heber (San Francisco, CA: ASP), 43

Rao, N. K., \& Lambert, D. L. 2010, in Recent Advances in Spectroscopy Theoretical, Astrophysical and Experimental Perspectives, ed. R. K. Chaudhuri, M. V. Mekkaden, A. V. Raveendran, \& A. Satya Narayanan (Berlin: Springer), 177

Rao, N. K., \& Nandy, K. 1986, MNRAS, 222, 357

Renzini, A. 1990, in ASP Conf. Ser. 11, Confrontation Between Stellar Pulsation and Evolution, ed. C. Cacciari \& G. Clementini (San Francisco, CA: ASP), 549

Robitaille, T. P., Whitney, B. A., Indebetouw, R., Wood, K., \& Denzmore, P. 2006, ApJS, 167, 256

Saio, H. 2008, in ASP Conf. Ser. 391, Hydrogen-Deficient Stars, ed. A. Werner \& T. Rauch (San Francisco, CA: ASP), 69

Schlegel, D. J., Finkbeiner, D. P., \& Davis, M. 1998, ApJ, 500, 525

Stanford, S. A., Clayton, G. C., Meade, M. R., et al. 1988, ApJ, 325, L9

Swinyard, B. M., Ade, P., Baluteau, J.-P., et al. 2010, A\&A, 518, L4

Tisserand, P., Wood, P. R., Marquette, J. B., et al. 2009, A\&A, 501, 985

Ueta, T., Meixner, M., \& Bobrowsky, M. 2000, ApJ, 528, 861

van Marle, A. J., Meliani, Z., Keppens, R., \& Decin, L. 2011, ApJ, 734, L26

Walker, H. J. 1985, A\&A, 152, 58

Walker, H. J. 1986, in IAU Colloq. 87, Hydrogen Deficient Stars and Related Objects, ed. K. Hunger, D. Schoenberner, \& N. Kameswara Rao (Astrophysics and Space Science Library, Vol. 128; Dordrecht: Reidel), 407

Walker, H. J. 1994, CCP7 News1., 21, 40

Webbink, R. F. 1984, ApJ, 277, 355

Weingartner, J. C., \& Draine, B. T. 2001, ApJ, 548, 296

Wheeler, J. C. 1978, ApJ, 225, 212

Whitney, B. A., Balm, S. P., \& Clayton, G. C. 1993, in ASP Conf. Ser. 45 Luminous High-Latitude Stars, ed. D. D. Sasselov (San Francisco, CA: ASP), 115

Whitney, B. A., Wood, K., Bjorkman, J. E., \& Cohen, M. 2003a, ApJ, 598, 1079

Whitney, B. A., Wood, K., Bjorkman, J. E., \& Wolff, M. J. 2003b, ApJ, 591, 1049

Whittet, D. C. B. 2003, Dust in the Galactic Environment (2nd ed.; Bristol: IOP)

Woitke, P., Goeres, A., \& Sedlmayr, E. 1996, A\&A, 313, 217

Wright, N. J., Barlow, M. J., Ercolano, B., \& Rauch, T. 2011, arXiv:1107.4554W 